\title{
Guidelines of Chinese Society of Clinical Oncology (CSCO) on Diagnosis and Treatment of Breast Cancer: an appraisal
}

\author{
Rani Chudasama, Mary Anne Fenton, Don S. Dizon \\ Division of Hematology-Oncology, Department of Medicine, The Warren Alpert Medical School of Brown University, Providence, RI, USA \\ Correspondence to: Don S. Dizon, MD. Rhode Island Hospital, 593 Eddy Street, Providence, RI 02903, USA. Email: don_dizon@brown.edu. \\ Comment on: Jiang Z, Song E, Wang X, et al. Guidelines of Chinese Society of Clinical Oncology (CSCO) on Diagnosis and Treatment of Breast \\ Cancer (2020 version). Transl Breast Cancer Res 2020. doi: 10.21037/tbcr-2020-2.
}

Received: 20 October 2020; Accepted: 27 October 2020; Published: 31 October 2020.

doi: $10.21037 /$ tbcr-2020-3

View this article at: http://dx.doi.org/10.21037/tbcr-2020-3

With 1.67 million new cases diagnosed in the world in 2012 , breast cancer is the most common cancer in women worldwide as well as the leading cause of cancer death. The highest incidence rates occur in the most developed regions of the world however mortality rates are higher in developing countries (1). Decreased survival rates in developing countries may be related to difficulty in access to early detection, diagnosis, and treatment of malignancy (2).

The predicted age-standardized incidence rate of breast cancer in China in 2012 was 22.1 per 100,000 person-years (1); however, this rate is felt to be rapidly increasing over the past decade as breast cancer is now the most frequently diagnosed female cancer in Chinese and Asian women. Studies have suggested that this rise in incidence could be related to decreased fertility rates, delayed age at first birth, decreased rates of breastfeeding and increasing body mass index $(3,4)$.

Given the increasing incidence of breast cancer in China, unified guidelines and recommendations regarding the diagnosis and treatment of breast cancer are needed. China is the world's largest developing country thus guidelines regarding breast cancer care should account for variability in socioeconomic status and access to care for patients. The Chinese Society of Clinical Oncology (CSCO) has put forth guidelines regarding breast cancer care that are evidence based and account for availability of resources in this population of patients (5).

Recommendations are categorized by grade based on criteria put forth to gage the characteristics of the evidence, which is in keeping with consensus guidelines in other countries, including the United States. The highest level of recommendation (grade 1) is based on large randomized controlled trials and should reflect unanimous consensus (defined as $\geq 80 \%$ expert agreement). The recommendations span from the initial evaluation and diagnosis of malignancy with radiologic imaging and pathologic work up, preoperative neoadjuvant treatment with chemotherapy and endocrine therapy through post-operative adjuvant treatment options. Complications from chemotherapy such as common side effects of nausea/vomiting, cardiotoxicity and bone marrow suppression are also addressed as well as treatment modalities for sites of metastatic disease.

One area of particular interest concerns the use of multigene profiling to guide clinical recommendations. Multiple platforms are currently available worldwide, but specific mention of the Recurrence Score (RS) is necessary. For women with hormone receptor-positive $\left(\mathrm{HR}^{+}\right)$disease, the results of the randomized TAILORRx clinical trial indicate that the RS is both prognostic for survival outcomes and can predict the benefits of treatment. In summary, these results indicate that women with a low (RS <11) to intermediate (RS 11-25) RS have an excellent prognosis with endocrine therapy alone; those with high RS (>25) benefit from adjuvant chemotherapy (6). As such, the RS has made its way into consensus guidelines for the management of early $\mathrm{HR}^{+}$breast cancer from multiple organizations, including the US-based National Comprehensive Cancer Network (NCCN) (7), the St. Gallen International Consensus Panel (8), and the European Society of Medical Oncology (ESMO) (9). In addition, multigene testing results are now incorporated into the American Joint Commission on Cancer (AJCC) (10) staging system for breast cancer, a nod to the importance of testing in the general management of breast cancer. 
These results, while acknowledged, do not make their way into a specific recommendation. Instead, the guidelines do not support multigene testing in all patients, but rather support a "rationally selected approach, based on differing risks". The rationale provided is the lack of data to evaluate the prognostic significance of these findings in Chinese patients. However, much of what we know has come from multi-institutional and often international clinical trials such that the findings, in the absence of society-specific data, can be universally applied. This is a particular departure from international consensus guidelines that could be reevaluated in future versions.

For patients with HER2-positive breast cancer, the guidelines strongly support the use of anti-HER2 directed therapy. For women who undergo neoadjuvant therapy, adjuvant recommendations are appropriately stratified by the pathologic response, drawing from international randomized trials to provide the basis for the guidelines. For the management of HER2-negative breast cancer, CSCO recommends the use of an anthracycline and a taxane (grade 1); platinum and taxanes are given a grade 2 recommendation, specifically for young patients with triplenegative breast cancer (TNBC), and particularly in the context of a confirmed BRCA mutation. The US NCCN takes a harder stance on the use of platinum, acknowledging it remains controversial in the neoadjuvant setting. Of note, the role of non-anthracycline neoadjuvant regimens is not discussed, such as docetaxel and cyclophosphamide (TC), which is listed as a "preferred regimen" in the US NCCN guidelines. There is some suggestion for incorporation of platinum agents in the neoadjuvant setting specifically for patients with TNBC for whom achieving better local control is necessary as several studies have shown improved pathologic complete response rates with use of platinum agents (11). However, there is little data regarding longterm outcomes and the optimal sequence of chemotherapy if platinum agents are used is not well-established. In addition, for patients with residual disease after neoadjuvant chemotherapy, specific guidelines are provided. Of note, capecitabine is offered as an adjuvant treatment specifically for those patients with TNBC who have residual disease, which is consistent with US NCCN recommendations. Finally, the role of neoadjuvant endocrine therapy is discussed, with recommendations on specific patients who are appropriate for this strategy as well as a precise follow-up strategy. This detail provides needed guidance to clinicians and expands on what is available elsewhere, for example, in the US NCCN recommendations.
The discussion on adjuvant therapy is comprehensive and is generally in line with international guidelines, drawing from randomized clinical trials as appropriate. It is noted that women diagnosed with breast cancer before 35 years fall in to a "lower risk of recurrence" stratum. This is interesting given that in Western cultures, young women often face a less than favorable prognosis compared to older women (12). Adjuvant endocrine therapy guidelines, including the definition of menopause, are also consistent with US guidelines. Of note, the role of ovarian function suppression (OFS) is specified, utilizing higher risk criteria in the role of OFS with tamoxifen or an aromatase inhibitor. The role of radiation therapy in the treatment of breast cancer is also extensively discussed.

The treatment approach to recurrent and metastatic breast cancer is comprehensive and details the use of newer agents, such as atezolizumab, and ones that are not available in the US, particularly bevacizumab. It is noted that novel agents that have shown to have activity are not yet included in these guidelines, including sacituzumab govitecan for TNBC, alpelisib for $\mathrm{HR}^{+}$breast cancer with a documented PIK3CA mutation, talazoparib for BRCA-associated breast cancer, larotrectinib or entrectinib for breast cancer associated with an NTRK fusion protein, the HER2-directed antibody-drug conjugates, fam-trastuzumab deruxtecan-nxki and tisotumab vedotin. These approvals in the US reflect the continuing evolution in breast cancer management and the rapid progress that has been made in treating these diseases.

Future recommendations regarding breast cancer care for patients in China could involve use of circulating tumor markers and next generations sequencing, however use of artificial intelligence (AI) is now currently recommended. CSCO expert group encourages the implementation of AIrelated clinical research as the CSCO team has been able to show that the IBM-system Watson for Oncology (WFO) has had good feasibility and effectiveness in decisionmaking for breast cancer treatment. In 2019, the CSCO AI system was officially released as a nationwide application that has increased the development of intelligent decisionmaking systems in China (13).

\section{Acknowledgments}

Funding: None.

\section{Footnote}

Provenance and Peer Review: This article was commissioned 
by the Editorial Office, Translational Breast Cancer Research. The article did not undergo external peer review.

Conflicts of Interest: All authors have completed the ICMJE uniform disclosure form (available at http://dx.doi. org/10.21037/tbcr-2020-3). The authors have no conflicts of interest to declare.

Ethical Statement: The authors are accountable for all aspects of the work in ensuring that questions related to the accuracy or integrity of any part of the work are appropriately investigated and resolved.

Open Access Statement: This is an Open Access article distributed in accordance with the Creative Commons Attribution-NonCommercial-NoDerivs 4.0 International License (CC BY-NC-ND 4.0), which permits the noncommercial replication and distribution of the article with the strict proviso that no changes or edits are made and the original work is properly cited (including links to both the formal publication through the relevant DOI and the license). See: https://creativecommons.org/licenses/by-nc-nd/4.0/.

\section{References}

1. Ferlay J, Soerjomataram I, Ervik M, et al. GLOBOCAN 2012 v1.0. Cancer Incidence and Mortality Worldwide. IARC Cancer Base No. 11 [internet]. Lyon, France: International Agency for Research on Cancer, 2013.

2. Sankaranarayanan R, Swaminathan R, Brenner H, et al. Cancer survival in Africa, Asia, and Central America: a population-based study. Lancet Oncol 2010;11:165-73.

3. Fan L, Strasser-Weippl K, Li JJ, et al. Breast cancer in China. Lancet Oncol 2014;15:e279-89.

4. Shin HR, Joubert C, Boniol M, et al. Recent trends and patterns in breast cancer incidence among Eastern and Southeastern Asian women. Cancer Causes Control

doi: $10.21037 /$ tbcr-2020-3

Cite this article as: Chudasama R, Fenton MA, Dizon DS. Guidelines of Chinese Society of Clinical Oncology (CSCO) on Diagnosis and Treatment of Breast Cancer: an appraisal. Transl Breast Cancer Res 2020;1:24.
2010;21:1777-85.

5. Jiang Z, Song E, Wang X, et al. Guidelines of Chinese Society of Clinical Oncology (CSCO) on Diagnosis and Treatment of Breast Cancer (2020 version). Transl Breast Cancer Res 2020. doi: 10.21037/tbcr-2020-2.

6. Sparano JA, Gray RJ, Makower DF, et al. Adjuvant Chemotherapy Guided by a 21-Gene Expression Assay in Breast Cancer. N Engl J Med 2018;379:111-21.

7. National Comprehensive Cancer Network Clinical Practice Guidelines in Oncology: Breast Cancer. V 6.2020, Sep. 8, 2020. Available online: https://www.nccn.org/ professionals/physician_gls/pdf/breast.pdf

8. Burstein HJ, Curigliano G, Loibl S, et al. Estimating the benefits of therapy for early-stage breast cancer: the St. Gallen International Consensus Guidelines for the primary therapy of early breast cancer 2019. Ann Oncol 2019;30:1541-57.

9. Cardoso F, Kyriakides S, Ohno S, et al. Early breast cancer: ESMO Clinical Practice Guidelines for diagnosis, treatment and follow-up†. Ann Oncol 2019;30:1194-220. Erratum in: Ann Oncol. 2019 Oct 1;30(10):1674. doi: 10.1093/annonc/mdz189. Ann Oncol. 2020 Sep 7:S09237534(20)42223-9. doi: 10.1016/j.annonc.2020.08.2158. Online ahead of print.

10. American Joint Committee on Cancer (AJCC) Staging Manual. 8th Edith. January 1, 2018.

11. Poggio F, Bruzzone M, Ceppi M, et al. Platinum-based neoadjuvant chemotherapy in triple-negative breast cancer: a systematic review and meta-analysis. Ann Oncol 2018;29:1497-508.

12. Radecka B, Litwiniuk M. Breast cancer in young women. Ginekol Pol 2016;87:659-63.

13. Li JB, Jiang ZF. Establishment and its application of Chinese society of clinical oncology artificial intelligence system (CSCO AI). Zhonghua Yi Xue Za Zhi 2020;100:411-5. 